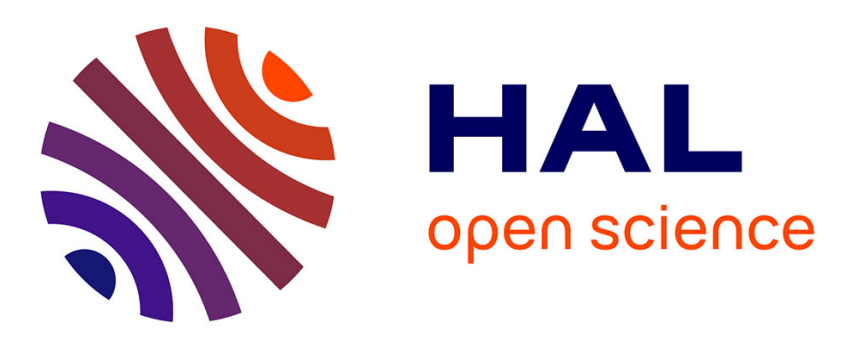

\title{
A step toward the Bermond-Thomassen conjecture about disjoint cycles in digraphs
}

Nicolas Lichiardopol, Attila Pór, Jean-Sébastien Sereni

\section{To cite this version:}

Nicolas Lichiardopol, Attila Pór, Jean-Sébastien Sereni. A step toward the Bermond-Thomassen conjecture about disjoint cycles in digraphs. SIAM Journal on Discrete Mathematics, 2009, 23 (2), pp.979-992. 10.1137/080715792 . hal-00487102

\section{HAL Id: hal-00487102 https://hal.science/hal-00487102}

Submitted on 27 May 2010

HAL is a multi-disciplinary open access archive for the deposit and dissemination of scientific research documents, whether they are published or not. The documents may come from teaching and research institutions in France or abroad, or from public or private research centers.
L'archive ouverte pluridisciplinaire HAL, est destinée au dépôt et à la diffusion de documents scientifiques de niveau recherche, publiés ou non, émanant des établissements d'enseignement et de recherche français ou étrangers, des laboratoires publics ou privés. 


\title{
A step towards the Bermond-Thomassen conjecture about disjoint cycles in digraphs
}

\author{
Nicolas Lichiardopol* $^{*}$ Attila Pór ${ }^{\dagger} \quad$ Jean-Sébastien Sereni ${ }^{\ddagger}$
}

\begin{abstract}
In 1981, Bermond and Thomassen conjectured that every digraph with minimum out-degree at least $2 k-1$ contains $k$ disjoint cycles. This conjecture is trivial for $k=1$, and was established for $k=2$ by Thomassen in 1983. We verify it for the next case, by proving that every digraph with minimum out-degree at least five contains three disjoint cycles. To show this, we improve Thomassen's result by proving that every digraph whose vertices have out-degree at least three, except at most two with out-degree two, indeed contains two disjoint cycles.
\end{abstract}

\section{Introduction}

Our notations mainly follow that of Bang-Jensen and Gutin [2]. By cycle we mean oriented cycle, that is an oriented path starting and ending at the same vertex. A cycle of length $d$ is a $d$-cycle. A 1-cycle is a loop and a 3-cycle is also called a triangle. All digraphs contained in this paper can have loops and 2-cycles but no parallel arcs. A digraph without cycles of length at most two is an oriented graph.

Fix a digraph $D=(V, A)$. Its order is the size of the vertex-set $V$. Given a subset $X$ of $V$, the sub-digraph of $D$ induced by $X$ is the digraph

*Lycée A. de Craponne, Rue Chateauredon, BP 55, 13651 Salon de Provence, France. E-mail: lichiar@club-internet.fr.

${ }^{\dagger}$ Department of Mathematics, Western Kentucky University, Bowling Green, KY 42101, USA. E-mail: attila. por@wku. edu. This author’s work was supported by the Hungarian National Foundation Grant T 046246.

${ }^{\ddagger}$ CNRS (LIAFA, Univsersité Denis Diderot), Paris, France, and Department of Applied Mathematics (KAM), Faculty of Mathematics and Physics, Charles University, Prague, Czech Republic. E-mail: sereni@kam.mff.cuni.cz. This author's work was supported by the European project IST FET AEOLus. 
$D[X]:=\left(X, A^{\prime}\right)$ where $A^{\prime}$ is the set of all arcs in $A$ that start and end in $X$. Two sub-digraphs $D_{1}$ and $D_{2}$ of $D$ are disjoint if their vertex-sets are. We write $v \rightarrow u$ to mean an arc from the vertex $v$ to the vertex $u$.

We let $D^{*}$ be the digraph obtained from $D$ by reversing the direction of every arc. For every vertex $v \in V$ let $N_{D}^{+}(v):=\{x \in V: v \rightarrow x \in A\}$ be the out-neighbourhood of $v$ in $D$, and let $d_{D}^{+}(v):=\left|N_{D}^{+}\right|$be the out-degree of $v$ in $D$. The vertices of $N_{D}^{+}(v)$ are the out-neighbours of $v$. The in-neighbourhood of $v$ in $D$ is $N_{D}^{-}(v):=N_{D^{*}}^{+}(v)$, and its in-degree is $d_{D}^{-}(v):=\left|N_{D}^{-}(v)\right|$. The vertices of $N_{D}^{-}(v)$ are the in-neighbours of $v$. If the context is clear, we may omit the subscript and just write $N^{+}(v)$ and $N^{-}(v)$.

Given two disjoint subsets $X, X^{\prime} \subset V$, the set $X$ dominates $X^{\prime}$ if $X^{\prime}$ is contained in the out-neighbourhood of each vertex of $X$. If the set $X$ is composed of only one vertex $v$ we simply say that $v$ dominates $X^{\prime}$. The set $X^{\prime}$ is dominated if there exists a vertex dominating it. The set $X$ dominates a sub-digraph $D^{\prime}$ of $D$ if it dominates its vertex-set $V\left(D^{\prime}\right)$.

An arc is $d$-dominated if it is dominated by a vertex of out-degree $d$.

We are interested in the following conjecture stated by Bermond and Thomassen in 1981.

Conjecture 1 ([3]). For every positive integer $k$, every digraph with minimum out-degree at least $2 k-1$ contains $k$ disjoint cycles.

It is an obvious observation if $k$ is one, and Thomassen gave a nice and simple proof of it when $k$ is two in 1983 .

Theorem 1 ([6]). Every digraph with minimum out-degree at least three contains two disjoint cycles.

Thomassen [6] also established the existence of a finite integer $f(k)$ such that every digraph of minimum out-degree at least $f(k)$ contains $k$ disjoint cycles. As noted by Bermond and Thomassen [3], such an integer cannot be less than $2 k-1$, so the bound offered by Conjecture 1 is optimal. Alon [1] proved that for every integer $k$, the value $64 k$ is suitable for $f(k)$ in 1996. Recently, Conjecture 1 has been verified for tournaments with minimum indegree at least $2 k-1[4,5]$.

Our main result is the following theorem, which proves Conjecture 1 when $k$ is three.

Theorem 2. Every digraph with minimum out-degree at least five contains three disjoint cycles.

We note that the method used by Alon [1] yields an upper bound on the order of a minimum counter-example to Conjecture 1. For instance, 
when $k$ is three the order of a minimum counter example is at most 42 . However, as pointed out by Alon [1], this bound is out of reach for a bruteforce attack. Thus we need to develop new tools to study this conjecture and prove Theorem 2. One of them is to strengthen Theorem 1.

Theorem 3. Let D be a digraph whose vertices have out-degree at least three, except at most two which have out-degree two. The digraph $D$ contains two disjoint cycles.

The paper is organised as follows. In the next section we slightly improve Thomassen's result by proving Theorem 3 which is a crucial ingredient in our proof of Theorem 2. Section 3 is devoted to the proof of a property of a certain class of digraphs, which may be of independent interest. In Section 4 we establish Theorem 2. The proof proceeds by contradiction: we consider a minimum counter-example $D$-with respect to the number of vertices - to the statement of the theorem, and exhibit some of its structural properties. Then, the argument is split into two cases: in Sub-section 4.1 we suppose that $D$ does not contain a triangle while in Sub-section 4.2 we establish the result if $D$ contains a triangle.

\section{Improving Theorem 1}

As mentioned earlier, Thomassen proved that Conjecture 1 is true if $k$ is two, namely every digraph with minimum out-degree three contains two disjoint cycles. The goal of this section is to strengthen this result, by proving Theorem 3.

Proof of Theorem 3. Contrary to the statement, let $D=(V, A)$ be a minimum counter-example with respect to the number of vertices. We also assume that each vertex has out-degree at most three. First, observe that $D$ cannot contain a loop. If $C$ is a loop, the digraph obtained from $D$ by removing the vertex of $C$ has minimum out-degree at least one, thus it contains a cycle $C^{\prime}$. The cycles $C$ and $C^{\prime}$ of $D$ are disjoint, a contradiction. So the order of $D$ is at least four. We now establish two properties of $D$. Recall that a sub-digraph is 2-dominated if there exists a vertex of out-degree two dominating it.

(A) Every 2-cycle of $D$ is 2-dominated. In particular $D$ contains at most two 2-cycles.

Suppose that $C:=u v$ is a 2 -cycle. Let $D^{\prime}$ be the digraph obtained from $D$ by removing $u$ and $v$. Then $D^{\prime}$ cannot have minimum out-degree at least one, otherwise it would contain a cycle which would be disjoint from $C$, a contradiction. Therefore there exists a vertex of $D$ of out-degree two 
dominating $C$, as asserted. From this fact it directly follows that $D$ does not contain more than two 2-cycles, since each vertex of out-degree two can dominate at most one 2 -cycle and $D$ contains at most two vertices of outdegree two.

The next property, proved by Thomassen [6], is still valid under our weaker assumptions.

(B) Every arc of $D$ is dominated.

Suppose that $u \rightarrow v \in A$ is not dominated. By Property $(A)$, we can assume that $v \rightarrow u$ is not an arc of $D$. Let $D^{\prime}$ be the digraph obtained from $D$ by first removing all arcs out-going from $u$ except $u \rightarrow v$, and then contracting the arc $u \rightarrow v$ into a new vertex $w$. The out-degree of $w$ in $D^{\prime}$ is equal to the out-degree of $v$ in $D$. Moreover, the out-degree of each other vertex of $D^{\prime}$ is the same as its out-degree in $D$. Hence, by the minimality of $D$, the digraph $D^{\prime}$ contains two disjoint cycles, which yield two disjoint cycles in $D$, a contradiction.

Fix a vertex $v$ and let $x$ be an in-neighbour of $v$. Note that $d_{D}^{-}(v) \geq 1$ by the minimality of $D$. As the $\operatorname{arc} x \rightarrow v$ is dominated, there exists a vertex $y \in V$ with $\{x, v\} \subseteq N^{+}(y)$. Consequently the digraph $D\left[N^{-}(v)\right]$ has in-degree at least one and thus contains a cycle. In particular the size of the in-neighbourhood of each vertex is at least two. Observe now that if $d_{D}^{-}(v) \geq 3$ for every $v \in V$, then $D$ indeed contains two disjoint cycles: just apply Theorem 1 to $D^{*}$.

Therefore, there exists a vertex of in-degree two in $D$, and hence a 2-cycle $C_{1}:=u v$. By Property $(A)$, let $z$ be a vertex of out-degree two dominating $u$ and $v$. The sub-digraph $D\left[N^{-}(z)\right]$ contains a cycle, which must intersect $C_{1}$. So we can assume that $u \rightarrow z \in A$, and we let $C_{2}$ be the cycle $z u$. Again by Property $(A)$, there exists a vertex $z^{\prime}$ of out-degree two that dominates $C_{2}$. Note that $z^{\prime} \neq v$, otherwise $D$ would contain three 2-cycles, thereby contradicting Property $(A)$. Observe also that neither $z$ nor $u$ can dominate $z^{\prime}$, otherwise $D$ would contain three 2 -cycles. Therefore the cycle contained in $D\left[N^{-}\left(z^{\prime}\right)\right]$ is disjoint from the 2-cycle $u z$, a contradiction. This contradiction concludes the proof.

We note that this result is optimal, since a symmetrically oriented triangle -i.e. three vertices $x_{1}, x_{2}, x_{3}$ with an arc from $x_{i}$ to $x_{j}$ whenever $i \neq$ $j$ - does not have two disjoint cycles. It is also optimal if we restrict ourselves to oriented graphs, since there exist oriented graphs on seven vertices with three vertices of out-degree two, four vertices of out-degree three and no two disjoint cycles. See Figure 1(a) for an example. Moreover, the oriented graph of Figure 1(b) has no two disjoint cycles, yet every vertex has out-degree three except one which has out-degree one. 


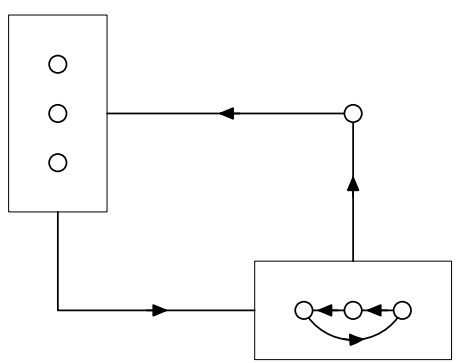

(a)

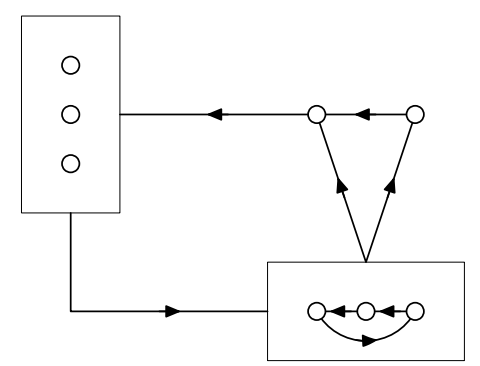

(b)

Figure 1: (a) An oriented graph with three vertices of out-degree two, four vertices of out-degree three and no two disjoint cycles, and $(b)$ an oriented graph whose vertices all have out-degree three, except one which has outdegree one, and yet without two disjoint cycles. An arc from/to a box goes from/to every vertex of the box.

\section{Arc-dominated oriented graphs}

We say that a digraph $D=(V, A)$ is arc-dominated if every arc of $A$ is dominated. As we will see, a minimum counter-example to Theorem 2 -and more generally, to Conjecture 1 -must be arc-dominated, and it must be an oriented graph-i.e. it contains neither a loop nor a 2-cycle. We put the following proposition in a dedicated section because we believe that it might be of independent interest.

Proposition 4. Let $D=(V, A)$ be an arc-dominated oriented graph, and let $X \subset V$ such that $D[X]$ is either acyclic or an induced cycle of $D$. There exists a cycle $C$ disjoint from $D[X]$ such that every vertex of $C$ has at least one out-neighbour in $X$.

Proof. We set $X^{\prime}:=V \backslash X$. Let $S$ be the set of vertices of $X^{\prime}$ having at least one out-neighbour in $X$. Observe that it is enough to prove that $D[S]$ contains a cycle. To this end, it suffices to establish that every vertex of $S$ has at least one in-neighbour in $S$. Suppose on the contrary that there exists a vertex $v \in S$ with no in-neighbour in $S$. We set $Y:=\left(N_{D}^{-}(v) \cup N_{D}^{+}(v)\right) \cap X$. By the definition of $S$, the vertex $v$ has an out-neighbour $x$ in $X$, so in particular $Y \neq \emptyset$. Since for every $y \in Y$ there is an arc between $v$ and $y$, and since $D$ is arc-dominated, there exists a vertex $z$ which dominates $\{v, y\}$. It follows that $z \in X \cap N_{D}^{-}(v) \subset Y$. In particular this proves that $D[Y]$ and hence $D[X]$ contains a cycle. This is not possible if $D[X]$ is acyclic and concludes the proof in this case. If $D[X]$ is an induced cycle $C^{\prime}$ of $D$, then $D[X]=D[Y]=C^{\prime}$. Consider the out-neighbour $y$ of $x$ in $C^{\prime}$. By what 
precedes, it is dominated by a vertex of $N_{D}^{-}(v) \cap X$, which must be $x$ since $C^{\prime}$ is induced. This is a contradiction since $\{v, x\}$ would induce a 2-cycle in D.

Corollary 5. Let $D=(V, A)$ be an arc-dominated oriented graph. Suppose that $C$ is a cycle of $D$, and $C^{\prime}$ an induced cycle disjoint from $C$. If there is no arc from a vertex of $C$ to a vertex of $C^{\prime}$ then $D$ contains three disjoint cycles.

Proof. We apply Proposition 4 with $X$ being $V\left(C^{\prime}\right)$. We deduce that there exists a cycle $C_{1}$ disjoint from $C^{\prime}$ such that every vertex of $C_{1}$ has an outneighbour in $C^{\prime}$. As there is no arc from $C$ to $C^{\prime}$, the cycle $C_{1}$ is certainly disjoint from $C$. Thus, $C, C^{\prime}$ and $C_{1}$ are three disjoint cycles of $D$.

\section{Proof of Theorem 2}

Our goal in this section is to establish Theorem 2. We proceed by contradiction: we suppose that the statement of the theorem is false, and consider a counter-example with the minimum number of vertices. We first establish some fundamental properties of such a digraph, which will be extensively used in the sequel. Until the end, we let $D=(V, A)$ be a counter-example to the statement of Theorem 2 with the smallest number of vertices, and subject to this with the smallest number of arcs. In particular, every vertex has out-degree exactly five. We let $n$ be the order of $D$. Note that $n \geq 5$.

Lemma 6. The following hold.

(i) The digraph $D$ is an oriented graph, i.e. it has no loop and no 2-cycle.

(ii) Every arc of $D$ is dominated. In particular, the in-neighbourhood of every vertex contains a cycle.

(iii) Every triangle of $D$ is dominated by three different vertices.

(iv) If a vertex $v$ dominates a cycle $C$, there exists a triangle vuw with $u \in V(C)$ and $w \notin V(C)$.

Proof. (i) Suppose that $C$ is a cycle of $D$ of length at most two. Note that the induced sub-digraph $D^{\prime}$ of $D$ obtained by removing the vertices of $C$ has minimum degree at least three. Thus, by Theorem $1, D^{\prime}$ contains two disjoint cycles, which are certainly disjoint from the cycle $C$. Hence, $D$ contains three disjoint cycles, a contradiction. 
(ii) It is proved exactly as Property $(B)$ in the proof of Theorem 3, so we do not repeat it here.

(iii) Let $C$ be a triangle of $D$, and consider the digraph $D^{\prime}$ obtained from $D$ by removing the vertices of $C$. The digraph $D^{\prime}$ has minimum out-degree at least two. Moreover every vertex of $D^{\prime}$ that does not dominate $C$ in $D$ has out-degree at least three in $D^{\prime}$. As $D^{\prime}$ cannot contain two disjoint cycles - otherwise $D$ would contain three disjoint cycles - the contrapositive of Theorem 3 implies that at least three vertices of $D^{\prime}$ have out-degree two, and hence these vertices dominate $C$ in $D$.

(iv) Let $C^{\prime}$ be an induced cycle contained in $N^{-}(v)$. As $v$ dominates a cycle $C$, by $(i)$ the cycles $C$ and $C^{\prime}$ are disjoint. According to Corollary 5 , there exists an arc from $C$ to $C^{\prime}$, which yields the sought triangle.

According to Item $(i)$ of the preceding lemma, $D$ is actually an oriented graph. So, as every vertex has out-degree five, we deduce that the order $n$ of $D$ is at least 11 . The proof is now split into two parts, regarding whether $D$ contains a triangle.

\subsection{The digraph $D$ does not contain a triangle}

In this sub-section, we assume that $D$ does not contain a triangle. In particular, every 4-cycle of $D$ is induced. We first establish some useful properties of $D$.

Lemma 7. For every vertex $v$ of $D$ the sub-digraph induced by the outneighbours of $v$ is acyclic.

Proof. Since $D$ has no triangle this follows directly by Lemma 6(iv).

We define a spanning sub-digraph $D^{\prime}$ of $D$ as follows. Recall that, by Lemma 6(ii), the in-neighbourhood of every vertex $u$ of $D$ contains an induced cycle $C_{u}$. We let $D^{\prime}=\left(V, A^{\prime}\right)$ be the spanning sub-digraph of $D$ where $A^{\prime}$ is comprised of all $\operatorname{arcs} v \rightarrow u$ of $D$ with $v \in V\left(C_{u}\right)$. The obtained digraph $D^{\prime}$ has some useful properties, stated in the next lemma.

Lemma 8. The following hold.

(i) If $v \rightarrow u$ belongs to $A^{\prime}$ then $N_{D}^{+}(v) \cap N_{D^{\prime}}^{-}(u) \neq \emptyset$.

(ii) The digraph $D^{\prime}$ is 4-regular, i.e. $d_{D^{\prime}}^{+}(v)=4=d_{D^{\prime}}^{-}(v)$ for every vertex $v$. In particular, $D$ contains a 4-cycle. 
(iii) If the arc $v \rightarrow u$ belongs to $A \backslash A^{\prime}$ then $N_{D}^{+}(v) \cap N_{D}^{-}(u)=\emptyset$.

Proof. (i) Let $v \in V\left(C_{u}\right)$. By the definition of $C_{u}$, the out-neighbour of $v$ in $C_{u}$ dominates $u$ in $D^{\prime}$ and belongs to $N_{D}^{+}(v)$.

(ii) By Lemma 6(ii), for every vertex $v$ we have $d_{D^{\prime}}^{-}(v) \geq 4$ since $D$ contains no triangle. Therefore, to prove the statement we only need to show that $d_{D^{\prime}}^{+}(v) \leq 4$ for every vertex $v$. Suppose on the contrary that $v$ is a vertex of $D$ with out-degree five in $D^{\prime}$. Hence, $N_{D}^{+}(v)=N_{D^{\prime}}^{+}(v)$. Let $u \in N_{D^{\prime}}^{+}(v)$. By $(i), N_{D^{\prime}}^{+}(v) \cap N_{D^{\prime}}^{-}(u) \neq \emptyset$. So the sub-digraph of $D^{\prime}$ induced by the out-neighbours of $v$ has minimum in-degree at least one, and hence it contains a cycle. This contradicts Lemma 7.

(iii) Suppose that $v \rightarrow u$ is an arc of $D$ contradicting the statement. Again, we shall prove that the out-neighbourhood of $v$ in $D$ contains a cycle, thereby contradicting Lemma 7. Let $z \in N_{D}^{+}(v)$, it suffices to prove that $z$ is dominated by a vertex of $N_{D}^{+}(v)$. If $z=u$ this is clear by the definition of $v$ and $u$, so suppose that $z \neq u$. By (ii), the vertex $v$ has out-degree four in $D^{\prime}$, thus $v \rightarrow z \in A^{\prime}$ and hence $(i)$ yields the conclusion.

We prove a last preliminary lemma before turning to the proof of Theorem 2.

Lemma 9. Let $C$ be a 4-cycle of $D$. The following hold.

(i) There exist at least three vertices with each exactly three out-neighbours in $C$;

(ii) at least one of the arcs of $C$ is not in $D^{\prime}$.

Proof. (i) By Lemma 7 every vertex of $D$ has at most three out-neighbours in $C$. Suppose that at most two vertices of $D$ have three out-neighbours in $C$. Then, every vertex of the sub-digraph of $D$ obtained by removing $C$ has out-degree at least three, except at most two vertices that have out-degree two. By Theorem 3, it contains two disjoint cycles. These two cycles together with $C$ yield three disjoint cycles in $D$, a contradiction.

(ii) Suppose on the contrary that $C:=x y z t$ is a 4-cycle of $D^{\prime}$. By the preceding item, there exist three vertices $a, b$ and $c$ with each three outneighbours in $C$. Note that no vertex of $C$ can dominate a vertex of $\{a, b, c\}$, otherwise $D$ would contain a triangle or a 2-cycle. As there are 
9 arcs from $\{a, b, c\}$ to $C$, at least one vertex of $C$, say $y$, is dominated by $\{a, b, c\}$. Furthermore, one of the arcs $a \rightarrow y, b \rightarrow y, c \rightarrow y$ is not in $D^{\prime}$. Otherwise, as $x \rightarrow y \in A^{\prime}$ and $d_{D^{\prime}}^{-}(y)=4$ by Lemma 8(ii), the cycle $C_{y}$ would be comprised of the vertices $a, b, c$ and $x$. This is not possible since there is no arc from $x$ to $\{a, b, c\}$. Without loss of generality we can assume that $a \rightarrow y \notin A^{\prime}$. By Lemma 8(iii), we deduce that the vertex $x$ is not an out-neighbour of $a$ in $D$. It follows that $a \rightarrow z$ and $a \rightarrow t$ are in $A$, and hence in $A^{\prime}$ by Lemma 8(iii) since $a$ dominates $\{y, z\}$.

We assert that $\{b, c\}$ dominates $\{x, t\}$ in $D$. By symmetry it is enough to prove that $b$ dominates $\{x, t\}$. If it is not the case then $b$ dominates $z$ in $D$. As $y \in N_{D}^{+}(b) \cap N_{D}^{-}(z)$, Lemma 8(iii) implies that $b \rightarrow z \in A^{\prime}$. Hence the induced cycle $C_{z}$ contains the vertices $a, y$ and $z$, which is a contradiction since $\{a, y\}$ dominates $z$. This proves the assertion.

Now, note that the arcs $b \rightarrow x$ and $c \rightarrow x$ must belong to $A^{\prime}$ by Lemma 8(iii). Consequently, the induced cycle $C_{x}$ contains the vertices $b, c$ and $t$, which is a contradiction since $\{b, c\}$ dominates $t$ in $D$. This concludes the proof.

We now switch to the proof of Theorem 2. We shall obtain a contradiction by proving that $D^{\prime}$ contains a 4-cycle. To this end, we first prove Property $(C)$ below, which states that $D$ contains a 4 -cycle with two consecutive arcs in $D^{\prime}$. As we shall see, this implies that $D^{\prime}$ contains a 4-cycle.

(C) There exists a 4-cycle of $D$ with two consecutive arcs belonging to $A^{\prime}$. By Lemmas $8(i i)$ and $9(i i)$, let $C:=x y z t$ be a 4 -cycle of $D$ with $x \notin V\left(C_{y}\right)$. Consequently, $C$ and $C_{y}$ are disjoint. Let us write $C_{y}=$ abcd with $a \notin V\left(C_{b}\right)$. So, the cycles $C_{y}$ and $C_{b}$ are disjoint. As $D$ does not have three disjoint cycles, we deduce that $C_{b}$ must contain a vertex of $C$. This vertex cannot be $x$, since by Lemma 8(iii) $x$ has no out-neighbour in $N_{D}^{-}(y)$. Moreover, it can be neither $y$ nor $z$-otherwise $D$ would contain a 2-cycle or a triangle. Hence $t \in V\left(C_{b}\right)$. The situation is depicted in Figure 2(a). Note that tbyz is a 4-cycle with two consecutive arcs in $D^{\prime}$, namely $t \rightarrow b$ and $b \rightarrow y$. This establishes Property $(C)$.

We are now in position to conclude the proof, by showing that there exists a 4-cycle of $D$ included in $D^{\prime}$ and thereby contradicting Lemma 8(ii). By Property $(C)$ let $C:=x y z t$ be a 4 -cycle of $D$ with two consecutive arcs in $D^{\prime}$. By Lemma $9(i i)$ at least one of the arcs of $C$ is not in $D^{\prime}$. Therefore, up to renaming the vertices, we can assume that $t \rightarrow x \in A, x \rightarrow y \in A$ and $z \rightarrow t \notin A^{\prime}$. Thus $C$ and $C_{t}:=a b c d$ are disjoint. By Lemma $9(i i)$, assume 


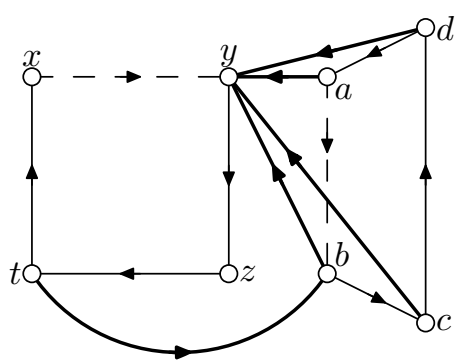

(a)

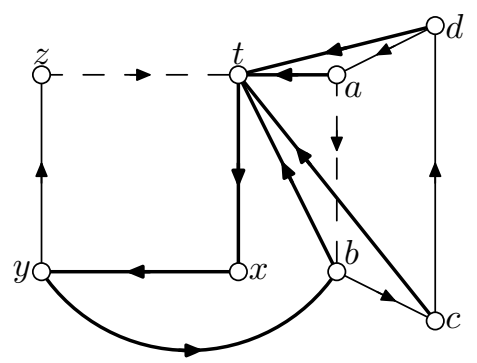

(b)

Figure 2: The arcs belonging to $A^{\prime}$ are drawn in bold, and the arcs not in $A^{\prime}$ are dashed. The remaining ones are only known to be in $A$.

that $a \notin C_{b}$. The cycles $C_{t}$ and $C_{b}$ being disjoint, $C_{b}$ must intersect the cycle $C$. As none of $x, z$ and $t$ has an out-neighbour in $C_{b}$, we infer that $y \in V\left(C_{b}\right)$. Therefore txyb is a 4-cycle of $D$ which is included in $D^{\prime}$, see Figure $2(b)$. This contradiction concludes the proof when $D$ does not contain a triangle.

\subsection{The digraph $D$ contains a triangle}

For every vertex $u \in V$, we let $\varphi(u)$ be the greatest integer $r$ for which there exist triangles $T_{1}, T_{2}, \ldots, T_{r}$ such that

- the intersection of every two triangles is the vertex $u$; and

- the in-neighbour of $u$ in $T_{i}$ dominates $T_{i-1}$ for every $i \in\{2,3, \ldots, r\}$.

Thus, $\varphi(u)=0$ if and only if $u$ is not contained in a triangle, and $1 \leq \varphi(u) \leq$ 5 otherwise.

Lemma 10. Either $D$ contains two disjoint triangles, or all the triangles of $D$ share a common vertex $x$. In the latter case $\varphi(x) \geq 3$.

Proof. Let $\Phi:=\max _{u \in V} \varphi(u)$. As $D$ contains a triangle, we deduce from Lemma 6(iii) and (iv) that $\Phi \geq 2$.

We suppose first that $\Phi=2$. We shall establish that $D$ contains two disjoint triangles. Suppose on the contrary that it is not the case. Then, the following holds.

(D) Every vertex $x \in V$ such that $\varphi(x)=2$ is dominated by a triangle.

By the definition of $\varphi$, there exist four vertices $y_{1}, y_{2}, z_{1}, z_{2}$ such that $T_{1}:=$ $x y_{1} z_{1}$ and $T_{2}:=x y_{2} z_{2}$ are two triangles and $z_{2}$ dominates $T_{1}$. According to Lemma $6(i i i)$, there exists a vertex $z_{3} \notin\left\{y_{1}, z_{1}\right\}$ dominating $T_{2}$. Thus, Lemma $6(i v)$ implies that there exists a triangle $T_{3}:=z_{3} a_{1} b_{1}$, with $a_{1} \in$ 


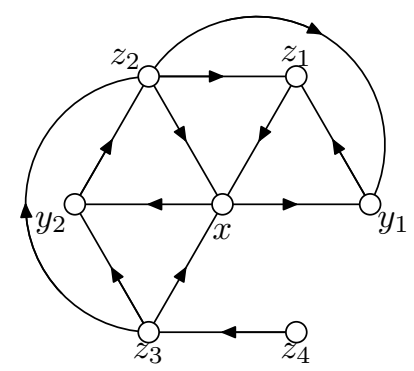

Figure 3: Configuration obtained when $\varphi(x)=2$.

$V\left(T_{2}\right)$ and $b_{1} \notin V\left(T_{2}\right)$. There are three distinct vertices that dominate $T_{3}$. Among the vertices so far defined, only $y_{1}$ and $z_{1}$ may dominate $T_{3}$. Thus, there exists $z_{4} \notin\left\{y_{1}, z_{1}\right\}$ that dominates $T_{3}$. Moreover, there exists a triangle $T_{4}:=z_{4} a_{2} b_{2}$ with $a_{2} \in V\left(T_{3}\right)$ and $b_{2} \notin V\left(T_{3}\right)$. The situation is depicted in Figure 3. We set $X:=\left\{x, y_{1}, z_{1}, y_{2}, z_{2}, z_{3}, z_{4}\right\}$.

If $z_{1} \rightarrow z_{3} \in A$, then $z_{3} z_{2} z_{1}$ is a triangle which dominates $x$, which would establish Property $(D)$. We thus assume in the remaining that $z_{1}$ does not dominate $z_{3}$. The vertex $b_{1}$ dominates $z_{3}$, thus either $b_{1}=y_{1}$ or $b_{1} \notin X$. We consider these two cases separately.

$b_{1} \notin X$. Then $a_{1}$ must be $x$, otherwise $z_{3} a_{1} b_{1}$ and one of $T_{1}, T_{2}$ are disjoint. Now, $T_{1}, T_{2}$ and $z_{3} x b_{1}$ show that $\varphi(x) \geq 3$, a contradiction.

$b_{1}=y_{1}$. Consider $T_{4}=z_{4} a_{2} b_{2}$. Note that $z_{4}$ dominates $b_{1}=y_{1}$. Notice also that the vertex $b_{2}$ does not lie in $\left\{y_{2}, z_{2}\right\}$, otherwise $z_{4} z_{3} b_{2}$ and $T_{1}$ would be two disjoint triangles. If $b_{2}=x$ then $T_{1}, T_{2}$ and $z_{4} z_{3} x$ show that $\varphi(x) \geq 3$, a contradiction. If $b_{2}=z_{1}$, then $z_{4} y_{1} z_{1}$ and $T_{2}$ are two disjoint triangles. Thus, as $b_{2} \neq b_{1}=y_{1}$ (since $b_{2} \notin V\left(T_{3}\right)$ ), we deduce that $b_{2} \notin X$. As $T_{4}$ must intersect $T_{1}, T_{2}$ and $T_{3}$, we infer that $a_{2}=x$. Consequently, $z_{3} z_{2} y_{1}$ and $T_{4}$ are two disjoint triangles, a contradiction.

This establishes Property $(D)$. Note that we also have showed that $z_{1}$ must indeed dominate $z_{3}$. Hence, $\varphi\left(z_{2}\right) \geq 2$, by considering the triangles $T_{2}$ and $z_{3} z_{2} z_{1}$.

Now consider a vertex $x$ such that $\varphi(x)=2$, and let $T_{1}$ and $T_{2}$ be two triangles as before. In particular, we can assume that the vertex $z_{2}$ satisfies $\varphi\left(z_{2}\right)=2$, thus is dominated by a triangle $T$. Observe that $T_{1}$ and $T$ are two disjoint triangles, a contradiction.

In conclusion, we have proved that $D$ contains two disjoint triangles if $\Phi$ is two.

We assume now that $\Phi \geq 3$, and we let $x$ be a vertex such that $\varphi(x)=\Phi$. By contradiction, suppose that $D$ does not contain two disjoint triangles, 


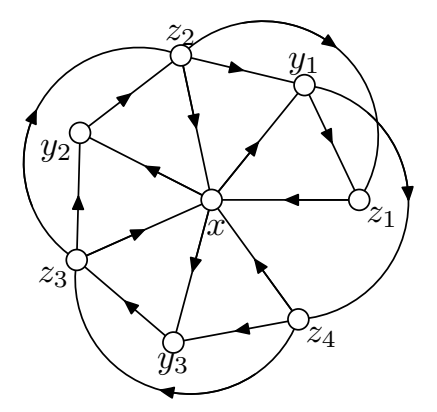

Figure 4: Configuration obtained when $\varphi(x) \geq 3$ and $b=y_{1}$.

and yet contains a triangle $T$ not containing $x$. There exist three triangles $T_{i}:=x y_{i} z_{i}, i \in\{1,2,3\}$, such that $V\left(T_{i}\right) \cap V\left(T_{j}\right)=\{x\}$ if $i \neq j$, and $z_{i}$ dominates $T_{i-1}$ if $i>1$. As $D$ does not contain two disjoint triangles, we deduce that $T$ contains a vertex from each set $\left\{y_{i}, z_{i}\right\}$, for $i \in\{1,2,3\}$.

According to Lemma 6(iii), there exists a vertex $z_{4}$, distinct from all the vertices defined so far, that dominates the triangle $T_{3}$. Thus, there exists a triangle $T_{4}:=z_{4} a b$, with $a \in V\left(T_{3}\right)$ and $b \notin V\left(T_{3}\right)$. Notice that $b \neq x$. Hence, if $a \neq x$, we obtain two disjoint triangles; indeed, the triangle $T_{4}$ intersects at most two triangles among $T_{1}, T_{2}$ and $T_{3}$, because $x \notin V\left(T_{4}\right)$ and $z_{4} \notin V\left(T_{1}\right) \cup V\left(T_{2}\right) \cup V\left(T_{3}\right)$. Thus, among the triangles $T_{i}, i \in\{1,2,3,4\}$, at least two are disjoint, a contradiction.

Therefore, $a=x$. Let $X:=\left\{x, y_{1}, z_{1}, y_{2}, z_{2}, y_{3}, z_{3}, z_{4}\right\}$. Note that $b$ either belongs to $\left\{y_{2}, y_{1}\right\}$ or does not belong to $X$. The latter case is not possible, since $T_{4}$ and $T$ would then be two disjoint triangles-because, as noted earlier, $V(T) \subset\left\{y_{1}, z_{1}, y_{2}, z_{2}, y_{3}, z_{3}\right\}$. If $b=y_{2}$, then $T_{1}$ and $z_{4} z_{3} y_{2}$ are two disjoint triangles. Therefore, we infer that $b$ is $y_{1}$, so $T_{4}=z_{4} x y_{1}$. The situation is depicted in Figure 4.

As $D$ does not contain two disjoint triangles, $V(T)$ must intersect the set $\left\{y_{1}, z_{4}\right\}$. So, $y_{1}$ is a vertex of $T$. Now, observe that the triangles $T_{2}, T_{3}$ and $T_{4}$ fulfil the same conditions as do $T_{1}, T_{2}$ and $T_{3}$. Consequently, we deduce as previously that $y_{2} \in V(T)$. So, the triangle $T$ either is $z_{3} y_{2} y_{1}$ or is comprised of the vertices $y_{1}, y_{2}$ and $y_{3}$. If the former case, let $u \notin X$ be a vertex dominating $T_{4}$. This is possible since at least three vertices dominate $T_{4}$. There exists a triangle $T_{5}$ comprised of $u$, a vertex $u_{1} \in V\left(T_{4}\right)$ and a vertex $u_{2} \notin V\left(T_{4}\right)$. If $u_{1} \in\left\{y_{1}, z_{4}\right\}$, then $T_{5}$ and either $T_{2}$ or $T_{3}$ are two disjoint triangles, since $x \notin V\left(T_{5}\right)$. So, $u_{1}=x$ and $u_{2}$ is either $y_{2}, y_{3}$ or a new vertex. In all cases, $T_{5}$ and $z_{3} z_{2} y_{1}$ are two disjoint triangles, a contradiction. Consequently, $V(T)=\left\{y_{1}, y_{2}, y_{3}\right\}$. Thus, none of the vertices $z_{i}, i \in\{1,2,3,4\}$, dominates $T$. As $T$ is dominated by at least three vertices, we can choose a vertex $u$ that dominates $T$ and is different from $x$. Now, 
there exists a triangle $T^{\prime}:=u u_{1} u_{2}$ with $u_{1} \in\left\{y_{1}, y_{2}, y_{3}\right\}$, and $u_{2} \notin V(T)$. Note that $u_{2} \neq x$. Consequently, $T^{\prime}$ and one triangle among $T_{1}, T_{2}$ and $T_{3}$ are disjoint, a contradiction. This concludes the proof.

We define now two subsets of $V$. Let $Y$ be the set of vertices contained in a triangle, and $Z$ the set of vertices dominating a triangle. We set $D_{Y}:=D[Y]$, and $D_{Z}:=D[Z]$. From Lemma $6(i v)$ we deduce that $D_{Z}$ is an induced subdigraph of $D_{Y}$. The following lemma will prove to be useful.

Lemma 11. The following hold.

(i) Every vertex of $Y$ has at least five in-neighbours in D, with at least four lying in $D_{Y}$;

(ii) the minimum in-degree of the digraph $D_{Z}$ is at least three.

Proof. (i) Let $T:=x y z$ be a triangle containing $x$. By Lemma 6(iii), there exist three vertices $u, v$ and $w$ that dominate $T$. By the definition of $Y$, the vertices $u, v, w$ and $z$, which are all in-neighbours of $x$, belong to $Y$. Thus, it only remains to show that there exists a fifth in-neighbour of $x$ in $D$. To this end, suppose on the contrary that $d_{D}^{-}(x)=4$. Consider the cycle $C_{x}$. Since $z$ is dominated by $\{u, v, w\}$, it cannot belong to $C_{x}$. Thus, $C_{x}$ is a triangle whose vertices are $u, v$ and $w$. In particular $T$ and $C_{x}$ are two disjoint cycles, and there is no arc from the triangle $T$ to the cycle $C_{x}$, which contradicts Corollary 5 .

(ii) Let $x$ be a vertex of $D_{Z}$. By Lemma $6(i v)$ there exists a triangle $T:=$ $x y z$, along with three vertices $u, v, w$ dominating $T$. Thus, $\{u, v, w\} \subseteq$ $N_{D_{Z}}^{-}(x)$, which proves the desired statement.

We finish the proof of Theorem 2 right after having established the following bound.

Lemma 12. Suppose that $T$ and $T^{\prime}$ are two disjoint triangles of $D$. If $\ell$ is the number of arcs between $T$ and $T^{\prime}$ then $n \leq 22-\ell$.

Proof. Let $X:=V(T) \cup V\left(T^{\prime}\right)$ and $X^{\prime}:=V \backslash X$. We shall obtain the desired inequality by counting the number $L$ of arcs from a vertex of $X$ to a vertex of $X^{\prime}$. Since every vertex has out-degree five, $L$ is $4 \times 6-\ell=24-\ell$. We now prove that $L \geq n+2$, which will imply that $n+2 \leq 24-\ell$, and hence $n \leq 22-\ell$. Note that every vertex of $X^{\prime}$ has an in-neighbour in $X$, otherwise $D$ would contain three disjoint cycles by Lemma 6(ii). As the digraph $D\left[X^{\prime}\right]$ is acyclic (and of order at least $n-6 \geq 5$ ), there exists a 
vertex $v \in X^{\prime}$ having no in-neighbour in $X^{\prime}$, and another vertex $w$ with at most one in-neighbour in $X^{\prime}$. All together, these two vertices have at least $3+2=5$ in-neighbours in $X$. Now, note that $T$ and $T^{\prime}$ are two disjoint triangles in $D_{Y}$. By Lemma $11(i), D_{Y}$ has minimum in-degree at least four - and so its order is at least nine. Consequently, there exists three vertices $a, b$ and $c$ of $Y \backslash X$ having at least four, three and two in-neighbours in $X$, respectively - otherwise $D_{Y}$, and hence $D$, would contain three disjoint cycles, a contradiction. According to Lemma 11(i), every vertex of $Y$ has in-degree at least five in $D$. If $\{v, w\} \subset Y$, we infer from what precedes that $L \geq 5+4+2+n-6-3=n+2$. If only one of $v, w$ lies in $Y$, we deduce that $L \geq 5+3+2+2+n-6-4=n+2$, while if none of them is in $Y$, we have $L \geq 3+2+4+3+2+n-6-5=n+3$.

We now obtain a contradiction by proving that $D$ indeed contains three disjoint cycles. Recall that the order of $D$ is at least 11. According to Lemma 10, either all the triangles of $D$ share a common vertex, or $D$ contains two disjoint triangles. We consider the two cases separately.

Case 1: $D$ does not contain two disjoint triangles. In this case, all triangles of $D$ share a common vertex, say $x$, and we have $\varphi(x) \geq 3$. All the vertices of $D_{Z}$ are in-neighbours of $x$, since $x$ is contained in every triangle. By Lemma 11(ii), the digraph $D_{Z}$ has minimum in-degree at least three. We assert that $D_{Z}$ has also minimum out-degree at least three. To see this, suppose the contrary, and let $z$ be a vertex with out-degree at most two in $D_{Z}$. Note that $x \notin Z$, so $z \neq x$. We set $D_{1}:=D_{Z}-z$. Observe that the digraph $D_{1}^{*}$ fulfils the hypothesis of Theorem 3 , since all its vertices have outdegree at least three (by Lemma 11(ii)) except at most two vertices which have out-degree two. Thus, the digraph $D_{1}^{*}$ contains two disjoint cycles. They yield two disjoint cycles of $D_{1}$, say $C_{1}$ and $C_{2}$. As $z \in D_{Z}$, there exists a triangle $T:=z u v$ in $D$. By the definition of $x$, we have $u=x$. As noticed earlier, $Z \subseteq N_{D}^{-}(x)$, hence the triangle $T$ is disjoint from both $C_{1}$ and $C_{2}$, a contradiction. Therefore $D_{Z}$ has minimum out-degree at least three. Let us set $m:=|Z|$. We shall lower bound $m$ as a function of $n$. As $D_{Z}$ has minimum out-degree three, every vertex of $Z$ has at least four out-neighbours in $Z \cup\{x\}$, and thus at most one in $Z^{\prime}:=V \backslash(Z \cup\{x\})$. So the following holds.

(E) The number of arcs from a vertex of $Z$ to a vertex of $Z^{\prime}$ is at most $m$.

Furthermore, by Theorem $1, D$ contains two disjoint cycles $C_{1}$ and $C_{2}$ comprised of vertices of $Z$. Observe that every vertex of $Z^{\prime}$ has at least one 
in-neighbour in $Z$ : otherwise, by Lemma $6(i i), D$ would contain a cycle comprised of vertices of $Z^{\prime} \cup\{x\}$, which together with $C_{1}$ and $C_{2}$ would yield three disjoint cycles, a contradiction. As $\varphi(x) \geq 3$, there exist three outneighbours $y_{1}, y_{2}$ and $y_{3}$ of $x$ in $Z^{\prime}$, each having at least three in-neighbours in $Z$, by Lemma 6(iii). Consequently, the following is true.

$(F)$ The number of arcs from a vertex of $Z$ to a vertex of $Z^{\prime}$ is at least $9+(n-1-m-3)=n-m+5$.

It follows from Properties $(E)$ and $(F)$ that

$$
2 m \geq n+5 .
$$

We now aim at bounding $|A|$, the number of $\operatorname{arcs}$ of $D$, in terms of $m$. Recall that $|A|=5 n$, since every vertex of $D$ has out-degree five. We partition $V$ into the sets $Z, Z^{\prime}$ and $\{x\}$. Recall that every vertex has in-degree at least three, by Lemma $6(i i)$. As $Z \subseteq Y$, each vertex of $Z$ has at least five in-neighbours in $D$ by Lemma $11(i i)$. So

$$
\sum_{v \in Z} d_{D}^{-}(v) \geq 5 m
$$

Recall also that $Z \subseteq N_{D}^{-}(x)$, thus

$$
\left|N_{D}^{-}(x)\right| \geq m
$$

Moreover, according to Lemma 11(i), every vertex of $Y$ has in-degree at least five in $D$, and $\left|Y \cap Z^{\prime}\right| \geq 3$ since $\varphi(x) \geq 3$. In particular, $x$ has at most two out-neighbours not in $Z$. As $x$ belongs to every triangle of $D$, every vertex not in $N_{D}^{+}(x)$ has in-degree at least four in $D$, by Lemma $6(i i)$. Therefore we obtain

$$
\sum_{v \in Z^{\prime}} d_{D}^{-}(v) \geq 3 \times 5+2 \times 3+(n-1-m-5) \times 4=4 n-4 m-3 .
$$

By Equations (2), (3) and (4), we infer that the number of arcs of $D$ is at least $5 m+m+4 n-4 m-3=4 n+2 m-3$. As $|A|=5 n$, we obtain

$$
2 m \leq n+3 .
$$

Equations (1) and (5) are contradictory, which concludes the first case of our proof.

Case 2: $D$ has two disjoint triangles. Let $T:=x y z$ and $T^{\prime}:=x^{\prime} y^{\prime} z^{\prime}$ be two disjoint triangles. Consider the sub-digraph $D_{1}$ of $D$ obtained by 


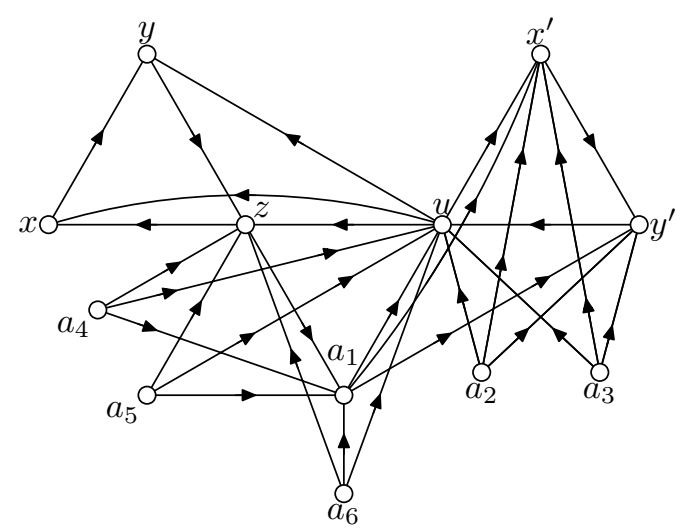

Figure 5: The sub-digraph $F$ of $D$.

removing $T$ and $T^{\prime}$. As $D$ does not contain three disjoint cycles, $D_{1}$ is acyclic, thus has a vertex $u$ of out-degree zero. Hence, the vertex $u$ has five out-neighbours among $x, y, z, x^{\prime}, y^{\prime}$ and $z^{\prime}$. Without loss of generality, let $N_{D}^{+}(u)=V(T) \cup\left\{x^{\prime}, z^{\prime}\right\}$. Necessarily, $y^{\prime} \in N_{D}^{-}(u)$, otherwise $T, T^{\prime}$ and $C_{u}$ would be three disjoint cycles of $D$, a contradiction. So $T$ and $T_{1}:=u x^{\prime} y^{\prime}$ are two disjoint triangles of $D$. By Lemma 6(iii), there exists an arc from a vertex of $T$ to a vertex of $T_{1}$. Moreover, there are at least three arcs from a vertex of $T_{1}$ to a vertex of $T$, since the vertex $u$ dominates $T$. So Lemma 12 implies that $n \leq 22-4=18$. By Lemma $6($ iii $)$, there exist three vertices $a_{1}, a_{2}$ and $a_{3}$ that dominate $T_{1}$. Clearly, none of these vertices belongs to $V(T) \cup V\left(T_{1}\right)$. Moreover at least one of them, say $a_{1}$, has no in-neighbour in $\left\{a_{1}, a_{2}, a_{3}\right\}$, since otherwise $T, T_{1}$ and $D\left[\left\{a_{1}, a_{2}, a_{3}\right\}\right]$ would be three disjoint cycles of $D$. By Lemma $6(i i)$, the vertex $a_{1}$ must have an in-neighbour in $T$, otherwise $T, T_{1}$ and $C_{a_{1}}$ would be disjoint, a contradiction. Without loss of generality, we assume that $z \in N_{D}^{-}\left(a_{1}\right)$. The triangle $T_{2}:=u z a_{1}$ is dominated by three vertices $a_{4}, a_{5}$ and $a_{6}$. Clearly, none of these vertices belongs to $V(T) \cup V\left(T_{1}\right) \cup\left\{a_{1}, a_{2}, a_{3}\right\}$. More precisely, among the vertices not in $T_{2}$, only $y^{\prime}, a_{2}$ and $a_{3}$ dominate $u$, and none of them dominates $a_{1}$. Thus, we obtain the sub-digraph $F$ of $D$, depicted in Figure 5. For convenience, every vertex of $D$ not in $F$ is called extern.

Note that all the vertices of $F$ belong to $Y$, and hence have in-degree at least five in $D$ by Lemma $11(i)$. As $D$ does not contain three disjoint cycles, there exists $i \in\{2,3, \ldots, 6\}$ such that the vertex $a_{i}$ does not have an in-neighbour in $\left\{a_{1}, a_{2}, \ldots, a_{6}\right\}$. Observe that a vertex dominating the arc $a_{i} \rightarrow u$ is either $y^{\prime}$ or extern, the former begin possible only if $i \geq 4$. We now consider two cases, regarding the value of $i$. 
$i \in\{2,3\}$. Without loss of generality, let $i=2$. The vertex $a_{2}$ has at least one in-neighbour in $T$, otherwise $T, T_{1}$ and $C_{a_{2}}$ would be three disjoint cycles of $D$. We consider two cases regarding whether $z$ dominates $a_{2}$.

$z$ dominates $a_{2}$. In this case, the triangle $z a_{2} u$ is dominated by three vertices, which must be extern. These three vertices belong to $Y$, as do the vertices of $F$. Thus, by Lemma 11(i), all have in-degree at least five in $D$. Furthermore, among them the vertex $u$ has in-degree at least 10 , and $z$ at least 8 . We deduce that

$$
|A|=5 n=\sum_{v \in V} d_{D}^{-}(v) \geq 10+8+13 \times 5+(n-15) \times 3,
$$

which implies that $n$ is at least 19 , a contradiction.

$z$ does not dominate $a_{2}$. So at least one vertex among $x, y$ dominates $a_{2}$. By symmetry of the roles played by $x$ and $y$ in what follows, we assume that $x$ dominates $a_{2}$. The triangle $T_{3}:=x a_{2} u$ is dominated by three vertices, which must be extern. These three vertices belong to $Z$, and hence to $Y$. The vertices of $F$ also belong to $Y$, and every vertex of $Y$ has in-degree at least five in $D$ by Lemma 11(i). Furthermore the in-degree of $u$ is at least 10 . Thus we obtain

$$
|A|=5 n \geq 10+14 \times 5+(n-15) \times 3,
$$

which yields $n \geq \frac{35}{2}$. As $n \leq 18$, we have $n=18$. Notice that $T$ is dominated by two vertices distinct from $u$. So, we infer that $d_{D}^{-}(x)+d_{D}^{-}(z) \geq 5+5+2=12$. Hence, we obtain

$$
|A|=5 n \geq 10+12+12 \times 5+(n-15) \times 3,
$$

from which it follows that $n \geq \frac{37}{2}$, a contradiction.

$i \in\{4,5,6\}$. Without loss of generality, let $i=4$. As $D$ does not have three disjoint cycles, $N_{D}^{-}\left(a_{4}\right) \cap\left\{x, y, x^{\prime}, y^{\prime}\right\} \neq \emptyset$. We split this case according to the corresponding sub-cases.

$x$ dominates $a_{4}$. We set $T_{3}:=a_{4} u x$. Among the vertices of $F$, only $y^{\prime}$ may dominate $T_{3}$. Supposing first that it is not the case, we obtain a contradiction by counting the number of $\operatorname{arcs}$ in $D$. The triangle $T_{3}$ is dominated by three extern vertices. These vertices belong to $Z$, and thus to $Y$. Moreover, recall that all the vertices of $F$ also belong to $Y$, and that every vertex of $Y$ has in-degree five, by 
Lemma $11(i)$. Thus, there are at least 15 vertices of in-degree five and, among them, $u$ has in-degree at least 10. Also, the vertex $z$ has in-degree at least 8 , because the triangle $x a_{4} z$ is dominated by three vertices, none of them lying in $\left\{y, u, a_{4}, a_{5}, a_{6}\right\}$. Therefore we obtain

$$
|A|=5 n \geq 10+8+13 \times 5+(n-15) \times 3,
$$

which yields $n \geq 19$, a contradiction.

Hence, the vertex $y^{\prime}$ dominates the triangle $T_{3}$. We seek a contradiction by counting the number of arcs in $D$. Note that there are at least five arcs between $T$ and $T_{1}$, since $u$ dominates $T_{1}, y^{\prime}$ dominates $x$ and there is at least one arc from $T$ to $T_{1}$ by Corollary 5 . So, by Lemma $12, n$ is at most 17 .

We now bound the number of $\operatorname{arcs}$ in $D$. As $a_{4}$ has no in-neighbours among the other vertices $a_{i}$, there exist two extern vertices dominating the triangle $T_{3}$. Recalling that all the vertices of $F$ belong to $Y$, we obtain $|Y| \geq 14$. By Lemma $11(i)$, each of these vertices has in-degree at least five in $D$. Moreover, $u$ has in-degree at least 9 , since it has already in-degree at least 7 in $F$. Also, the in-degree of $z$ is at least 8 , because $z$ is dominated by $\left\{u, a_{4}, a_{5}, a_{6}, y\right\}$, and by the three vertices dominating the triangle $a_{4} z x$, which cannot be any of the preceding ones. Therefore we infer that

$$
|A|=5 n \geq 9+8+12 \times 5+(n-14) \times 3,
$$

and hence $n \geq \frac{35}{2}$, contradicting the conclusion of the preceding paragraph.

$y$ dominates $a_{4}$. Let $T_{3}:=a_{4} u y$. This triangle is dominated by three vertices. Among the vertices of $F$, only $y^{\prime}$ may dominate it. Suppose first that it is not the case, i.e. $T_{3}$ is dominated by three extern vertices, which hence belong to $Y$. Furthermore, the triangle $T$ is dominated by two vertices different from $u$. Thus we deduce that $d_{D}^{-}(y)+d_{D}^{-}(z) \geq 5+5+2=12$. Note also that $u$ has in-degree at least 10 . So, recalling that all the vertices of $F$ belong to $Y$, it follows that

$$
|A|=5 n \geq 10+12+12 \times 5+(n-15) \times 3,
$$

i.e. $n \geq \frac{37}{2}$, a contradiction. Consequently, we infer that $y^{\prime}$ dominates $T_{3}$. As in the previous case, we note that there are at least five $\operatorname{arcs}$ between $T$ and $T_{1}$, and thus the Lemma 12 implies that 
$n$ is at most 17 . As $T_{3}$ is dominated by two extern vertices, notice that $u$ has in-degree at least 9 (since its in-degree in $F$ is at least 7). Moreover the triangle $a_{4} a_{1} y^{\prime}$ is dominated by three vertices, and none of them belongs to $\left\{x^{\prime}, a_{2}, a_{3}, a_{5}, a_{6}, z\right\}$. Hence, we deduce that both $a_{1}$ and $y^{\prime}$ have in-degree at least 7 in $D$. Therefore, we obtain

$$
|A|=5 n \geq 9+7+7+11 \times 5+(n-14) \times 3,
$$

so $n \geq 18$, a contradiction.

$x^{\prime}$ dominates $a_{4}$. Then the triangle $T_{3}:=a_{4} u x^{\prime}$ is dominated by three extern vertices. So there are at least 15 vertices of in-degree at least five, and among them $u$ has in-degree at least 10 (since its in-degree in $F$ is at least 7 ), and $x^{\prime}$ has in-degree at least 7 (since its in-degree in $F$ is at least 4$)$. Therefore, we deduce that

$$
|A|=5 n \geq 10+7+13 \times 5+(n-15) \times 3,
$$

which yields $n \geq \frac{37}{2}$, a contradiction.

None of $x, y$ and $x^{\prime}$ dominates $a_{4}$ in $D$. In this case the vertex $y^{\prime}$ must dominate $a_{4}$. We consider three vertices dominating the triangle $T_{3}:=a_{4} a_{1} y^{\prime}$. Among the vertices of $F$, only $x$ and $y$ can dominate $T_{3}$, but none of them does since none of them is an in-neighbour of $a_{4}$. Thus, $T_{3}$ is dominated by three extern vertices. Consequently, $Y$ contains at least 15 vertices, and $u, a_{1}$ and $y^{\prime}$ all have in-degree at least 7 . It follows that

$$
|A|=5 n \geq 3 \times 7+12 \times 5+(n-15) \times 3,
$$

and hence, $n \geq 18$. As we know that $n \leq 18$, we have $n=18$. In particular, there are exactly 6 extern vertices $r, s, t, r^{\prime}, s^{\prime}$ and $t^{\prime}$, with $\{r, s, t\}$ dominating the triangle $T_{3}$.

Now observe that, for every $i \in\{1,2,3,4\}, a_{i} \notin N_{D}^{+}\left(x^{\prime}\right)$. Moreover, $V(T) \cap N_{D}^{+}\left(x^{\prime}\right)=\emptyset$ otherwise there would be at least five arcs between $T$ and $T^{\prime}$, which would imply that $n \leq 17$ by Lemma 12 , a contradiction. We assert that the in-degree of $x^{\prime}$ in $D$ is at least 7. Recalling that $u, a_{1}$ and $y^{\prime}$ also have in-degree at least 7 , we would deduce that

$$
|A|=5 n=90 \geq 4 \times 7+11 \times 5+3 \times 3=92,
$$

a contradiction. So it only remains to prove the assertion. If $\left\{a_{5}, a_{6}\right\} \cap N_{D}^{+}\left(x^{\prime}\right) \neq \emptyset$, we assume without loss of generality that 
$x^{\prime}$ dominates $a_{5}$. Then, the triangle $a_{5} a_{1} x^{\prime}$ is dominated by three vertices, which cannot be any of $u, a_{1}, a_{2}, a_{3}$. So $x^{\prime}$ has at least 7 in-neighbours in $D$. If $\left\{a_{5}, a_{6}\right\} \cap N_{D}^{+}\left(x^{\prime}\right)=\emptyset$, the vertex $x^{\prime}$ has at least four out-neighbours lying in $\left\{r, s, t, r^{\prime}, s^{\prime}, t^{\prime}\right\}$. So it dominates at least one of $r, s$ and $t$, say $r$. The triangle $r a_{1} x^{\prime}$ is dominated by three vertices, none of them lying in $\left\{u, a_{1}, a_{2}, a_{3}\right\}$. Thus, we again conclude that the vertex $x^{\prime}$ has in-degree at least 7 , which proves the assertion.

The proof of Theorem 2 is complete.

\section{References}

[1] N. Alon. Disjoint directed cycles. J. Combin. Theory Ser. B, 68(2):167178, 1996.

[2] J. Bang-Jensen and G. Gutin. Digraphs. Springer Monographs in Mathematics. Springer-Verlag London Ltd., London, 2001. Theory, algorithms and applications.

[3] J.-C. Bermond and C. Thomassen. Cycles in digraphs - a survey. J. Graph Theory, 5(1):1-43, 1981.

[4] S. Bessy, N. Lichiardopol, and J.-S. Sereni. Two proofs of the BermondThomassen conjecture for tournaments with bounded in-degree. Discrete Mathematics, Sixth Czech-Slovak International Symposium on Combinatorics, Graph Theory, Algorithms and Applications. To appear.

[5] S. Bessy, N. Lichiardopol, and J.-S. Sereni. Two proofs of BermondThomassen conjecture for regular tournaments. In Proceedings of the sixth Czech-Slovak International Symposium on Combinatorics, Graph Theory, Algorithms and Applications, volume 28 of Electronic Notes in Discrete Mathematics, pages 47-53. Elsevier, 2007.

[6] C. Thomassen. Disjoint cycles in digraphs. Combinatorica, 3(3-4):393396, 1983. 